\title{
Expression and significance of HLA-DR/CD14 in peripheral blood mononuclear cells of critical patients in ICU.
}

\author{
Lijing Jia ${ }^{1}$, Lili Wang ${ }^{1}$, Yanan Wang ${ }^{1}$, Heng Zhang ${ }^{1}$, Da Ji', Wang Hong Xin ${ }^{2}$, Wei Chen ${ }^{\text {* }}$ \\ ${ }^{1}$ Emergency Department, the General Hospital of the People's Liberation Army, PR China \\ ${ }^{2}$ Department of Emergency, Affiliated Hospital of Logistical College of Chinese People's Armed Police Forces, Tianjin, \\ PR China
}

\begin{abstract}
Objective: To observe and explore the expression rate of HLA-DR/CD14 in peripheral blood monocytes of ICU patients and its clinical significance.

Methods: From October 2015 to April 2015 hospitalized patients with 60 cases, including ICU in critically ill patients (group A), 30 cases (group B) 30 patients with other diseases and 30 healthy physical examination to the same period as the healthy control group, the application of flow cytometry to detect the peripheral blood mononuclear cell surface the HLA-DR/CD14 expression rate, and the determination of research group A C-reactive protein (CRP) level, the analysis of CRP and HLA-DR/ CD14 expression rate.

Results: The study group A HLA-DR/CD14 expression rate (36.93+13.26)\% was significantly lower than the group $B(55.48 \pm 20.38) \%$ and healthy control group $(75.84 \pm 7.65) \%, P<0.01$ difference have statistical significance, and research group $B$ was significantly lower than that of healthy controls $(P<0.05)$. Correlation analysis showed that the expression rate of HLA-DR/CD14 in ICU patients was negatively correlated with CRP level $(\mathrm{r}=-\mathbf{0 . 6 8 9}, \mathrm{P}<0.01)$.

Conclusion: Peripheral blood mononuclear cell surface HLA-DR/CD14 expression rate is closely related to the severity of infection and disease, and can be used as a judgment of ICU critical illness severity and prognosis of immune index, worthy of clinical use.
\end{abstract}

Keywords: ICU, Expectations, Peripheral blood monocytes, HLA-DR/CD14.

Accepted on November 23, 2017

\section{Introduction}

The main task of intensive care medicine is to rescue the dying patients. With the aging of population and rapid progress in a variety of medical techniques, the intensive care medicine plays an increasingly important role in modern medicine [1]. Working in the ICU, we are faced with ever-changing challenges, including the emerging infectious diseases, drugresistant bacteria, patients with immunodeficient disorder caused by various treatment and patients with severe trauma [2]. In addition to professional medical issues, medical staffs in the ICU are facing the challenges of legal ethics, and how to communicate with anxious families and face the challenges of disease together is important [3]. Infectious disease is an important factor threatening the human health and longevity for a long time, and sepsis refers to the systemic response caused by infection. Infectious disease will lead to inflammation, necrosis and other related lesions in infected organs, and the host will produce immune response against the pathogen to eliminate the disease and restore the health. These reactions are known as systemic inflammatory response syndrome (SIRS) [4]. A large number of studies have shown that the occurrence of sepsis and MOLDS after severe trauma and burn is closely related to the immune function disorder [5]. A total of 60 patients admitted from October 2015 to April 2017 were selected, including 30 critical patients in ICU (Group A) and 30 patients with other diseases (Group B); 30 healthy subjects were selected during the same period as healthy control group. The human leukocyte antigen DR (HLA-DR)/cluster of differentiation 14 (CD14) expression rate on the surface of peripheral blood mononuclear cells was detected, and the effect was significant. It is now reported as follows.

\section{Clinical Data and Methods}

\section{General data}

Total 60 patients, admitted from October 2015 to April 2017 were selected. There were 30 critical patients in ICU (Group A), including 23 males and 7 females aged 20-78 years old with an average of $(54.9 \pm 5.2)$ years old. According to the diagnostic criteria recommended by American College of Chest Physicians/Society of Critical Care Medicine (ACCP/ 
SCCM), they were diagnosed with sepsis. The severity of disease was assessed using the Acute Physiology and Chronic Health Evaluation (APACHE) II score. There were 30 patients with other diseases (Group B), including 22 males and 8 females aged 26-80 years old with an average of $(56.7 \pm 6.3)$ years old. At the same time, 30 healthy subjects were selected during the same period as the healthy control group, including 20 males and 10 females aged $21-77$ years old with an average of $(53.2 \pm 5.9)$. The general data had no significant differences between the two groups of patients $(\mathrm{P}>0.05)$.

\section{Detection method of HLA-DR/CD14}

Detecting instruments: HLA-DR/CD14+\% was measured using a flow cytometer (BD, USA). The expression rate of HLA-DR/CD14 on the surface of peripheral blood mononuclear cells in each group was detected.

Preparation of analytical solution: Within $6 \mathrm{~h}$ after patients were diagnosed with sepsis in the ICU, $3 \mathrm{~mL}$ venous blood was extracted sterilely and treated with anti-coagulation using ethylenediamine tetraacetic acid disodium salt, followed by centrifugation using a centrifugal machine to separate the serum, used for subsequent PCR and CRP detection. $100 \mu \mathrm{L}$ anticoagulant blood was taken and placed into the detection and control tubes; $20 \mu \mathrm{L}$ CD14-PE and $20 \mu \mathrm{L}$ HLA-DR-FITC were added into the detection tube, while $20 \mu \mathrm{L}$ mouse CD14$\mathrm{PE}$ and $20 \mu \mathrm{L} \mathrm{IgG1-FITC}$ were added into the control tube, respectively. The solution in the four tubes was mixed for reaction in a dark place at room temperature for $15 \mathrm{~min}$. Then $2 \mathrm{~mL}$ red blood cell lysate was added for reaction for $10 \mathrm{~min}$, and the mixture was centrifuged using a centrifugal machine at $1000 \mathrm{rpm}$ for $5 \mathrm{~min}$. After centrifugation, the supernatant was discarded, and $2 \mathrm{~mL}$ PBS was added into each tube, followed by centrifugation for $5 \mathrm{~min}$ under the original parameters. Then the supernatant was discarded, finally $1 \mathrm{~mL}$ QIAzol analytical solution was added and mixed into the tube, and then the mixture was stored in a refrigerator at $-80^{\circ} \mathrm{C}$ for standby application, followed by RT-PCR, CRP and WBC detection on the machine.

HLA-DRT expression in CD14: The expression was detected via flow cytometry. The cell surface was stained and incubated in a dark place at room temperature for $30 \mathrm{~min}$. Then the HLADR expression in CD14 was measured using a flow cytometer (BD, USA). The data were obtained and analyzed using FACS Diva software.

RT-PCR detection: The QIAzol analysis reagent (QIAGEN) was used according to the instructions to extract the HLADRT/CD14 in peripheral blood in analytical solution. The RNA was isolated and the serum PCT was detected via sandwich immunoassay method and fluorescence detection method; PCT $>0.50 \mu \mathrm{g} / \mathrm{L}$ indicated the positive.

CRP level detection: The CRP level was detected via scatter turbidimetry using the full-automatic biochemical analyzer (Roche Group). CRP $>8.0 \mathrm{mg} / \mathrm{L}$ indicated the positive. The level in Group A was detected, and the relationship between CRP and HLA-DR/CD14 expression rate was analyzed.
WBC detection: WBC in whole blood was detected via whole blood and flow cytometry. WBC $>12 \times 10^{9} / \mathrm{L}$ indicated the positive. The full-automatic blood cell analyzer and its supporting reagents (SYSMEX, Japan) were used.

\section{Statistical processing}

SPSS23.0 statistical software package was used for the statistical treatment of HLA-DR/CD14 expression rate and CRP level in the three groups. All measurement data were presented as mean \pm standard deviation, and independentsamples t-test was used for the intergroup comparison. Enumeration data were presented as cases and percentage, and chi-square test was used for the intergroup comparison. $\mathrm{P}<0.05$ suggested that the difference was statistically significant.

\section{Results}

\section{Comparison of HLA-DR/CD14 expression rate among three groups of patients}

The expression rate of HLA-DR/CD14 in Group A [(36.93 \pm $13.26) \%$ ] was significantly lower than those in Group B $[(55.48 \pm 20.38) \%]$ and healthy control group $[(75.84 \pm$ $7.65) \%]$, and the differences were statistically significant $(\mathrm{P}<0.01)$. Besides, the expression rate in Group $\mathrm{B}$ was significantly lower than that in healthy control group $(\mathrm{P}<0.05)$. Correlation analyses showed that the expression rate of HLADR/CD14 in Group A was negatively correlated with the CRP level $(\mathrm{r}=-0.689, \mathrm{P}<0.01)$ (Tables 1 and 2$)$.

\section{Correlation analysis between HLA-DR/CD14 and ICU stay}

Spearman correlation analysis showed that HLA-DR/CD14 was negatively correlated with ICU stay $(\mathrm{r}=-0.482, \mathrm{P}=0.004)$, not correlated with total hospital stay $(\mathrm{r}=0.226, \mathrm{P}=0.257)$, and negatively correlated with APACHE II $(\mathrm{r}=-0.326, \mathrm{P}=0.000$, Figure 1).

Table 1. Comparisons of HLA-DR/CD14 expression rate and CRP level among three groups of patients.

\begin{tabular}{llll}
\hline Group & $\mathbf{n}$ & HLA-DR/CD14 (\%) & CRP (mg/L) \\
\hline Group A & 30 & $36.93 \pm 13.26$ & $37.28 \pm 12.52$ \\
\hline Group B & 30 & $55.48 \pm 20.38$ & $6.47 \pm 2.57$ \\
\hline Healthy control group & 30 & $75.84 \pm 7.65$ & $3.21 \pm 1.82$ \\
\hline F value & & 10.382 & 8.317 \\
\hline P value & 0.000 & 0.008 \\
\hline
\end{tabular}

Table 2. Comparisons of PCT and WBC detection results among three groups of patients.

\begin{tabular}{llll}
\hline Group & $\mathbf{n}$ & PCT level $(\mathbf{n g} / \mathrm{mL})$ & WBC level (109/L) \\
\hline A group & 30 & $24.83 \pm 16.72$ & $22.63 \pm 8.12^{\#}$ \\
\hline B group & 30 & $0.11 \pm 0.09$ & $6.02 \pm 1.38^{*}$ \\
\hline
\end{tabular}




\begin{tabular}{l}
\hline C group $\quad 30 \quad 0.01 \pm 0.00$ \\
\hline $\begin{array}{l}\text { Note: \# In the comparison of Group A and Group C, } P<0.01 ;{ }^{*} \text { In the comparison } \\
\text { of Group B and Group C, } P<0.01 .\end{array}$
\end{tabular}
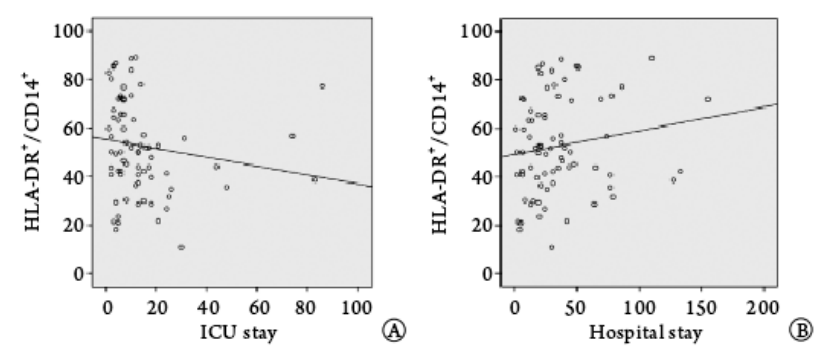

Figure 1a. Correlations of HLA-DR/CD14 with ICU stay (A) and total hospital stay (B) in Group A.

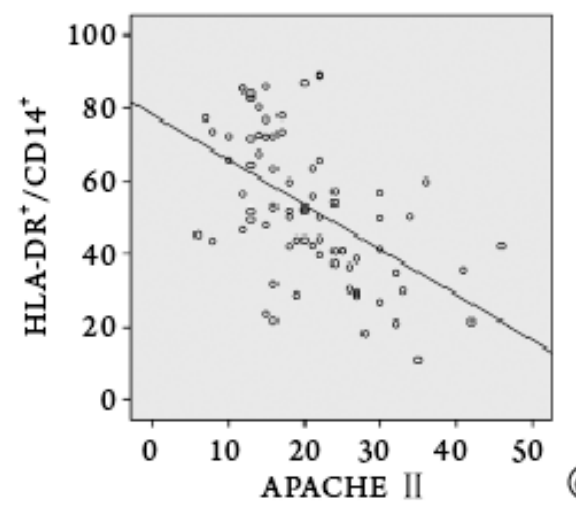

Figure 1b. Correlation between HLA-DR/CD14 and APACHE II in Group A.

\section{Discussion}

Sepsis is the systemic response of host to pathogen infection, which has always been a major disease that threatens the human health and life [6,7]. Severe sepsis is often complicated with organ failure, so the life of these patients is threatened and they need more active medical care in the ICU, so sepsis has been the main issue of intensive care medicine [8]. In the organ failure of severe sepsis patients, respiratory failure is the most common cause. Sepsis patients with respiratory failure often require the respirator therapy. The disease in these patients often develops into acute respiratory distress syndrome, facing a serious threat to life; and sepsis is also one of the most important causes of acute respiratory distress syndrome. Some sepsis patients will suffer from decline in blood pressure, and if the average arterial pressure is still less than $65 \mathrm{mmHg}$ after infusion therapy, application of booster, it is the so-called septic shock. These operational definitions related to sepsis aim to define the varied disease objectively, assist the clinical diagnosis and treatment, and facilitate the teaching and research. However, this definition is still unsatisfactory for the clinicians and researchers, because the sensitivity and specificity of definition of systemic inflammatory response syndrome are insufficient, and it stresses that the inflammation and severe sepsis, even septic shock, are not continuous phenomena. Joerg pointed out that the severe trauma can cause changes in the body environment, and induce the synthesis and release of pro-inflammatory factors and anti-inflammatory media, forming the network and waterfall effect. Excessive inflammatory response causes serious damage to the body, and leads to the immune dysfunction, among which monocyte/ macrophage system plays a key role in the identification of pathogenic microorganisms and their products, initiation of signal transduction and regulation of immune response.

In the infection and other inflammatory response states, the liver will synthesize acute-stage CRP. This indicator is an important basis for the immune response, and the concentration higher than $40-100 \mathrm{mg} / \mathrm{L}$ suggests the serious infection or sepsis in patients. When severe sepsis occurs, the CRP value usually reaches $200-500 \mathrm{mg} / \mathrm{L}$. In this study, the CRP level in sepsis group was significantly higher than those in the other two groups, indicating a positive correlation between CRP and sepsis. However, the sensitivity and specificity of CRP in the diagnosis of sepsis or septic shock are only $78.6 \%$ and $55.2 \%$, and CRP has a relatively non-specificity, which can be elevated in other non-bacterial diseases. This study indicated that CRP is also increased in other diseases, and CRP cannot be used as an accurate indicator of stress identification when diagnosing such patients, so it is weakly correlated with the severity of sepsis, and cannot be used as an accurate indicator of clinical diagnosis and treatment of sepsis. PCT will be significant increased in the bacterial infection state and when the infection is complicated, so it cannot be used as a precise indicator for clinical research. WBC will also be significantly increased in case of microbial infection, which is affected by a variety of factors, such as patient's age, type of infection, physiological function, immunity and overreaction; all these factors will affect the indicators, so WBC lacks accuracy in the early diagnosis of sepsis.

CD14, as a marker of monocyte, neutrophil and macrophage, is specific, which is a member in the cell surface glycoprotein family, as well as a leukocyte surface differentiation antigen found the earliest and a lipopolysaccharide cell receptor, affecting the onset and development body immune response, infectious diseases, epigenetic diseases and multiple systemic diseases. Under the physiological state, CD14 is a monocyte/ macrophage differentiation marker, whose transcription level is regulated by myeloid cell differentiation. Under the pathological state, CD14 can be used as an index in the prognosis evaluation and clinical guidance based on the abnormal CD14-specific expression in specific parts of mononuclear/macrophage and abnormal serum Scd14 concentration. Monocyte-macrophage system plays an important role in human non-specific immunity with phagocytic and antigen-presenting functions, while monocyte HLA-DR is directly related to the antigen-presenting ability of monocyte-macrophage to lymphocyte, so the detection of HLA-DR expression on the surface of peripheral blood mononuclear cells can reflect the immune function of the body and contribute to the diagnosis and prognosis of clinical infectious diseases. The relevant results showed that the HLA$\mathrm{DR} / \mathrm{CD} 14$ expression rate can reflect the immunosuppressive status of patients with infectious diseases, especially acute 
symptoms, and can be used as a sensitive and effective indicator for predicting prognosis and guiding immunostimulation. Moreover, the dynamic observation of HLA-DR/CD14 expression rate can detect the development of disease and correctly evaluate the prognosis. In this study, the expression rates of HLA-DR/CD14 in ICU patients, patients with other diseases and healthy subjects were compared. The results revealed that the expression rate of HLA-DR/CD14 in ICU patients $[(36.93 \pm 13.26) \%]$ was significantly lower than those in patients with other diseases $[(55.48 \pm 20.38) \%]$ and healthy control group $[(75.84 \pm 7.65) \%]$; the expression rate in patients with other diseases was obviously lower than that in healthy control group. Correlation analysis showed that the expression rate of HLA-DR/CD14 in ICU patients was negatively correlated with CRP level $(\mathrm{r}=-0.689, \mathrm{P}<0.01)$. Therefore, the expression rate of HLA-DR/CD14 on the surface of peripheral blood mononuclear cells is closely related to the severity of infection and disease, so it can be used as an immune index to judge the severity and prognosis of ICU acute and severe diseases, which is worthy of clinical promotion and application.

\section{References}

1. Li M, Li QW, Wu XG, Li YQ. Effects of berberine in different concentrations on inducing differentiation and maturation of monocyte-derived dendritic cells. Shandong Med J 2017; 57: 27-30.

2. Wu YH, Qi Y, Ma WK, Li YZ, Yang WG, Li CR. Expression of miR-146a in CD (14)+ Monocytes in Children with Sepsis and its Clinical Significance. J Clin Med Pract 2017; 21: 119-122.
3. Hu YF, Wang CM, Song YF, Wei Y. Clinical significance of HLA-DR expression rate in CD14 positive monocytes in elderly patients with severe pneumonia. Clin Med Eng 2017; 24: 26-27.

4. Lu JF, Tong HS, Chen RL, Shi XZ, Yin HM, Li Y, Zhang M, Su L. Prognosis analysis of traumatic sepsis in elderly patients via peripheral blood CD14+ monocyte HLA-DR. Med J Chinese PLA 2016; 41: 570-574.

5. Hu XL, Yao YG, Wu RZ, Liu SX, Zhao ZG, Clinical application of monocyte human leukocyte antigen DR in predicting severe multiple trauma infection. Chinese J Nosocomiol 2016; 26: 456- 458.

6. Li LJ, Chen W, Gu XY. Evaluation of severity of sepsis via dynamic changes in plasma PCT and CRP levels and its correlation. Chinese J Lab Diagnosis 2013; 17: 1010-1013.

7. Zhong YF, Lei ZY, Guo XM. Diagnostic value of combined detection of PCT, hs-CRP and WBC in the diagnosis of infection type and severity of sepsis in children. Med Rev 2015; 2015: 3631-3634.

8. Xiong XM, Li W. Clinical significance of HLA-DR/CD14 in peripheral blood mononuclear cells and its correlation with procalcitonin, C-reactive protein and white blood cell count. Lett Biotechnol 2014; 25: 394-397.

\section{*Correspondence to}

\section{Wei Chen}

Emergency Department

The General Hospital of the People's Liberation Army

PR China 